Itinéraires Itinéraires

Littérature, textes, cultures

\title{
Corps dessinés, corps créés, signés « Tom of Finland »
}

\section{Guy Snaith}

\section{(2) OpenEdition}

1 Journals

Édition électronique

URL : http://journals.openedition.org/itineraires/364

DOI : 10.4000/itineraires.364

ISSN : 2427-920X

Éditeur

Pléiade

\section{Édition imprimée}

Date de publication : 1 mai 2009

Pagination : 108-128

ISBN : 978-2-296-08444-5

ISSN : 2100-1340

\section{Référence électronique}

Guy Snaith, "Corps dessinés, corps créés, signés « Tom of Finland » , Itinéraires [En ligne], 2009-1 | 2009, mis en ligne le 13 juin 2014, consulté le 10 décembre 2020. URL : http://

journals.openedition.org/itineraires/364; DOI : https://doi.org/10.4000/itineraires.364

Ce document a été généré automatiquement le 10 décembre 2020.

\section{(c) $($ () $\odot$}

Itinéraires est mis à disposition selon les termes de la licence Creative Commons Attribution - Pas d'Utilisation Commerciale - Pas de Modification 4.0 International. 


\title{
Corps dessinés, corps créés, signés «Tom of Finland »
}

\author{
Guy Snaith
}

1 Durant une bonne partie de sa vie, Touko Laaksonen a travaillé pour une agence de publicité américaine à Helsinki, d'abord comme dessinateur, ensuite comme cadre ${ }^{1}$. C'est ce qu'il faisait pendant la journée. Le soir, pourtant, on le trouvait dans son appartement, vêtu de cuir, devant une feuille blanche, un crayon à la main, ses photos à proximité. Depuis les années 1950, Touko menait deux carrières, l'une respectable, pendant la journée, et l'autre secrète, la nuit, à travailler à ses "dessins cochons ${ }^{2}$ ". C'est grâce à ces dessins, signés "Tom of Finland", que les attributs d'une certaine masculinité sont devenus la propriété d'hommes qui, jusque-là, s'étaient vu priver de tout droit à la virilité. Le couronnement de la carrière de Tom of Finland a été de se voir attribuer le mérite d'avoir créé le prototype de l'homosexuel masculin de la fin du xx siècle.

\section{Un problème corporel}

Pour Michel Foucault, l'oppression de l'homosexualité date de sa médicalisation, voire de sa pathologisation à la fin du XIX e siècle (Foucault 1996 : 387). Depuis ce moment, la société hétéronormative exige que tout soit clairement classé, surtout tout ce qui est déviant. Selon Lee Edelman, au $x^{\mathrm{e}}$ siècle l'idéologie hétérosexiste a insisté sur «la nécessité de "lire" le corps en tant que signifiant de l'orientation sexuelle ${ }^{3}$ " (Edelman 2009 : 135). Ayant valeur de norme, l'hétérosexualité, puisqu'elle est naturelle et authentique, n'a pas besoin d'être lue. Mais pour discerner ce qui est anormal, il faut pouvoir reconnaître ce qui diffère de la norme. C'est le service que l'efféminé rendait à la société hétérosexuelle : c'est à travers l'efféminé que le corps homosexuel se trouvait marqué par "un code anatomique distinct, et donc lisible ${ }^{4}$ (Edelman 2009: 136). L'homosexualité inscrite sur le corps le rendait lisible comme texte. Pour Edelman donc, l'homosexualité a « une relation déterminante à la textualité et la lisibilité des signes $^{5} »$ (Edelman 2009: 139). En encourageant la masculinisation du corps 
homosexuel, Tom effaçait les traces de l'efféminé avec pour résultat qu'il n'était plus possible pour les hétérosexuels de lire le corps gay. Il restait pourtant possible pour les homosexuels, avec leur savoir d'initiés, de décoder les signes. Et c'est là précisément que réside le côté insidieux de l'homosexualité, et sa menace : elle peut rester invisible si l'on ne dispose pas de connaissances spécifiques.

Pour fonctionner, la société a besoin d'ordre ; il est donc nécessaire qu'elle dispose les choses, selon un ordre décidé en fonction des intérêts de la majorité, sans en considérer les limites ou les défauts. Richard Dyer a montré que les stéréotypes ont un rôle important à jouer dans la création d'un tel ordre. Ils constituent des raccourcis, dont l'efficacité réside dans le fait que le stéréotype exprime le consensus général sur un groupe social, consensus qui révèle aussi le siège du pouvoir dans telle ou telle société. Dyer constate que la fonction la plus importante du stéréotype est de maintenir des frontières claires, de définir qui est admissible et qui inadmissible: "Le rôle du stéréotype est de rendre visible l'invisible, pour qu'il n'y ait aucun danger qu'il ne nous prenne au dépourvu ${ }^{6}$ » (Dyer 1993: 16). Tom déclare la guerre au stéréotype de l'efféminé imposé aux homosexuels par une société férue d'ordre. Il refuse l'idée que les homosexuels constituent un troisième sexe indéterminé, moitié homme moitié femme. Il cherche à bannir les sentiments de honte qu'éprouvaient beaucoup d'homosexuels à l'époque de sa jeunesse. Par ses dessins, il a voulu corriger les injustices, donner confiance, inculquer la fierté et encourager les hommes comme lui. Tom confirme l'affirmation de Foucault pour qui la résistance, étant aussi le fruit de l'oppression, n'est pas seulement négative mais constitue en outre un processus créateur. Et Foucault partage avec lui un certain optimisme: "Nous avons toujours des possibilités, il y a toujours des possibilités de changer la situation ${ }^{7} »$ (Foucault $1996: 386$ ).

\section{Une solution corporelle}

Pour Tom, la façon de changer la situation sera la virilisation de l'homosexualité, les outils seront le crayon et le papier et la réalisation de son projet prendra, au cours d'une carrière d'une quarantaine d'années, la forme de plus de trois mille dessins et bandes dessinées peuplés par des caractères hypermasculins tels que Pekka et Kake, ainsi que des centaines d'hommes sans nom. Pour combattre le stéréotype, en inscrivant la virilité sur le corps des hommes dans ses dessins, il offrira ce qu'il appelle un prototype, un "meilleur exemplaire », un idéal de masculinité. Il serait surpris de constater que, déjà à la fin des années 1970, beaucoup d'homosexuels, surtout en Amérique du Nord, avaient réussi à suivre l'exemple de ses dessins pour se transformer en « Tom's Men ", mais c'est dans la nature du prototype d'être « le premier exemplaire d'un modèle, construit avant la fabrication en série", selon la définition minimale qu'en donne Le Petit Robert. Pour Tom, corpographèse signifie donc des stratégies de dessin et d'inscription du corps masculin ainsi que des signes non verbaux comme les gestes et les postures, le tout dans le but de renverser les codes établis et d'en établir de nouveaux, lesquels sont cette fois créés par un homme gay et doivent contribuer à l'élaboration d'une nouvelle identité sexuelle pour toute une génération d'homosexuels euro-américains. Le projet de catégorisation par le corps est éternel, pourtant, mais maintenant c'est le corps en forme et en muscles qu'il faut lire pour déterminer la sexualité. 
Quelles stratégies Touko a-t-il donc adoptées dans ses dessins pour valoriser ceux qui sont attirés par leur semblable? Quand il s'agit de la virilisation de l'homosexualité, il faut d'abord avoir une image de ce qui est masculin. Étant donné ses jeunes années passées à la campagne, l'image masculine que Touko avait devant les yeux n'était pas celle de son père instituteur, mais plutôt de types d'hommes qui travaillaient de leurs mains : ouvriers agricoles, bûcherons, routiers, conducteurs d'autobus... Et pendant la Seconde Guerre mondiale, une fois le couvre-feu instauré, soldats et marins étaient prêts à tout. Le jeune Touko associait alors la virilité à des hommes qui travaillaient à des tâches manuelles, ou à des militaires. À force de travailler comme bûcheron ou comme ouvrier agricole, le corps masculin se muscle. Une musculature puissante étant une particularité naturelle de l'homme, les muscles sont ainsi métaphoriques du pouvoir, de l'autorité, de la force, de la domination, qualités masculines "naturelles » que la société hétéronormative déniait à l'homosexuel. Après la guerre, pourtant, en Finlande comme ailleurs, tout est redevenu "normal », c'est-à-dire que les militaires sont retournés à leur caserne ou à leur pays d'origine, et les homosexuels à leurs gestes efféminés, leurs petits rires bêtes et leur maquillage. Pour s'intégrer à ce retour au monde homosexuel d'avant-guerre, Touko a essayé d'adopter ce type de comportement, mais peu à l'aise avec le côté « folle » de la vie homosexuelle, il l'a vite abandonné, réagissant contre le stéréotype qui associait l'homosexuel à l'efféminé8. Ses dessins des années 1940 célèbrent la beauté des jeunes plutôt que le corps musclé, mais au début des années 1950 un ami lui montre un magazine américain de culture physique. C'est comme un coup de foudre: Touko est tellement impressionné par les photos de jeunes gens en parfaite forme physique et portant seulement un petit cachesexe, que cela lui inspire l'idée de créer des dessins basés sur les corps des modèles qu'il voyait sur les photos. Même s'il déclare: "Je voulais les rendre aussi naturels que possible ${ }^{9}$ ", un portrait fidèle de ces jeunes athlètes ne lui suffit pas. Dès le début, en les copiant, Touko les dote d'attributs physiques particulièrement développés, améliorant l'original. En 1957, le premier dessin qu'il vend à Bob Mizer pour un de ces magazines américains de culture physique, Physique Pictorial, est l'image d'un jeune bûcheron torse nu se tenant en équilibre sur un rondin en plein milieu d'une rivière ${ }^{10}$. Tout au long de sa carrière, Tom restera fidèle aux hommes qu'il avait connus en grandissant ou pendant la guerre, et qui étaient pour la plupart des prolétaires: soldats, marins, policiers, gendarmes, pompiers, routiers, cow-boys, réparateurs, garagistes, maîtresnageurs, gardes forestiers, ouvriers du bâtiment, travailleurs manuels ou travailleurs de force. Ce sont des hommes actifs, dont la vie se passe au grand air, et dont les occupations sont celles d'un "vrai mec». Ce sont aussi pour la plupart des hommes contemporains et non pas campés à l'antique comme on en voyait chez d'autres artistes érotiques de l'époque. James Williams le souligne : «Croyant qu'un homme pouvait être fort, heureux, viril ET homosexuel, il commença à formuler le prototype qui est devenu la quintessence de la masculinité gay dans le monde entier ${ }^{11}$. (Williams 1989 : 35)

6 C'est ainsi par un processus d'exagération que Tom s'est mis à créer ses prototypes. Il l'affirme lui-même, tout en signalant comment cela faisait partie de son projet de masculinisation de l'homosexualité : «Bientôt je me mis à exagérer sciemment leur masculinité pour démontrer que tous ceux qui sont gay ne sont pas obligés d'être "ces sacrées folles", qu'ils pouvaient être aussi beaux, forts et masculins que n'importe quel autre homme ${ }^{12}$ " (dans Ramakers 2000: 65). Tom gardait des albums remplis de coupures de modèles ou de motifs à incorporer dans ses dessins. Plus tard, il a utilisé des modèles vivants qu'il photographiait lui-même, travaillant ensuite à partir de la 
photographie, une technique qu'il appelait "photoréaliste ${ }^{13}$ ». Au moyen d'un crayon bien taillé, Tom cherchait une netteté d'image, une clarté de ligne et de contour, qui ressembleraient à la photographie. De temps en temps il dessinait aussi au pastel, mais il n'aimait jamais autant le résultat, préférant une finition très achevée, où la main de l'artiste ne se voit pas. Berndt Arell appelle les années 1970 et 1980 « une période de virtuosité technique ", pendant laquelle Tom atteint une "précision [...] presque magique ", qui rend difficile de savoir s'il s'agit d'une photo ou d'un dessin ${ }^{14}$ (Arell et Mustola 2006 : 105). Ce sont aussi les décennies où l'on pourrait mettre en question l'emploi de naturel pour caractériser ses hommes. Mais Tom confesse que dès les années 1960 on lui demandait des images d'hommes de plus en plus exagérées ${ }^{15}$. Il faisait aussi concurrence à la photographie et voulait montrer plus que l'appareil photographique ne le pourrait. Toutes choses qui ont mené à une période d'exagération et de mensurations hypertrophiées.

7 En exagérant la musculature des modèles sur les photos, Tom modifie le corps masculin même, pour créer un corps encore plus imposant, aux épaules carrées, aux pectoraux énormes, aux biceps saillants, aux cuisses puissantes, aux fesses musclées. L'importance que Tom accorde aux muscles a pour résultat que le type corporel qu'on associe le plus avec lui est celui du culturiste musclé. Brian Pronger appelle les muscles « des symboles puissants de la masculinité16 ", ajoutant que les pectoraux surtout occupent un rang élevé parmi les signes masculins (Pronger 1990: 154-157). Comme dit Dyer: "Les muscles sont le signe du pouvoir - naturel, réalisé, phallique ${ }^{17} »$ (Dyer 1992: 205). L'avantage qu'ont ses hommes est que chez Tom le pénis aussi bien que les muscles est imposant. S'agit-il de pénis en fait? "Le phallus est le pénis qui vous coupe le souffle [...] par sa majesté18 » (Bordo 1999: 87), pénis qu'on considère avec une révérence craintive, comme maints hommes dans les dessins de Tom. Pronger cite Thorkil Vanggard qui appelle le phallus «une représentation picturale de l'essence de la virilité, représentation de la synthèse de tout aspect imaginable de la virilité véritable ${ }^{19}$ »(Pronger $1990: 159)$. Et, plus il est grand, plus il incarne le pouvoir et la puissance en tant que "signe ultime de la masculinité20 ${ }^{20}$ (Pronger 1990 : 161). Il va sans dire que «cette apothéose des symboles masculins ${ }^{21}$ » (160) fera partie du projet de Tom, mais dans ses dessins, il ne s'agit pas seulement de pénis énormes, parce que, chez Tom, par la dureté des muscles du torse et l'exagération des extrémités - les grosses mains, les poings serrés, les pieds bottés -, le corps entier est phalliquement codé. Dutton relève « le symbolisme bien établi qui associe le corps dur et musclé avec le phallus engorgé, en érection et puissant ${ }^{22}$ » (Dutton $1995:$ 236). Dans les dessins où ses hommes se tiennent debout, seuls, sans fond contre la blancheur du papier, inébranlables, carrés d'épaules, durs comme le roc, Tom transforme le corps en métaphore phallique. De plus, ses hommes portent des phallus seuls ou ailés comme logo ou sigle sur leur casquette, ou leurs manches, ou le col de leur chemise, ce qui donne une spécificité au monde créé par Tom. Chez Tom le phallus devient un métonyme, symbolique de son œuvre et de ses hommes. Tomland est phallocentrique.

8 Le corps masculin est sa matière première, son argile, son canevas : la musculation, le pénis, la peau, les cheveux, les poils, les gestes, le regard. Au long d'une carrière d'une quarantaine d'années, Tom exploite impitoyablement tout ce que le corps masculin peut supporter : il l'exagère, l'altère, le transforme en le gonflant, le rasant, le perçant, le polissant ou le salissant; il écrit même dessus, en le faisant tatouer. En fait, les premières pages du livre Tom of Finland. The Art of Pleasure présentent une image par décennie de la vie créatrice de Tom, des années 1940 aux années 1980 : les années des 
folles, des bûcherons, des motards, des clones, des fétichistes. Pour chaque image, des flèches montrent comment lire l'image parce que, pour Tom, le corps est signifiant comme texte. Prenons par exemple le motard qui représente les années 1960. On lit les indications suivantes :

1. approche de la trentaine, 2. porte des bottes de motard, 3. se met à fréquenter les salles de musculation, 4. n'oublie pas ses pectoraux, 5. s'élargit le tour de taille, 6. s'est fait pousser la frange, 7. et les pattes, 8. est très poilu, 9. développe ses tétons, 10. porte une casquette en cuir souple, 11. avec un insigne phallique, 12. fume, 13. aime les tee-shirts blancs et moulants, 14. ne sort jamais sans son blouson de cuir, 15. vit sans quitter son jean, 16. muni d'une braguette à boutons, bien entendu !, 17. a perdu sa ceinture, 18. commence à faire craquer les coutures [la flèche désigne le pénis que la braguette a des difficultés à contenir], 19. porte l'inscription « enculeur » au dos de son blouson (on ne sait jamais), 20. fréquente les bars [on lui offre un verre], 21. devinez ce qu'il cherche, 22. sourit moins [que dans les dessins des années 1950]. (Tom of Finland 1998)

9 Alors, le corps entier est érotisé, et toute communication se fait par des signes non verbaux. Même dans ses histoires de Kake, une série de bandes dessinées érotiques dans laquelle le héros est un jeune homme d'allure finlandaise qui s'appelle Kake (c'està-dire "macho"), on n'est pas loquace; les paroles sont minimales, l'histoire se communiquant au lecteur presque exclusivement par le dessin et entre les personnages par les regards et les gestes, les bulles étant absentes. Dans le film Daddy, Tom explique que son public étant international, on ne pouvait pas inclure de texte. Il fallait utiliser le visage et les gestes comme moyens de communication entre ses personnages. Il est donc commode que la testostérone semble rendre les hommes plus sensibles à ce qui est visuel que les femmes (Bordo, 1999 : 170). Et on pense au cliché selon lequel le silence est masculin et la parole, féminine.

10 Le look que Tom recherche doit communiquer l'autorité et le pouvoir. Le visage est donc sévère; le sourire n'étant pas sexy, on ne sourit pas; les yeux sont graves, le regard pénétrant. Les hommes de Tom se présentent à leur public en tant que spectacle sexuel. Ils ont été créés pour être regardés mais ils ne tombent pas dans le binarisme qui associe le regard avec l'activité et le fait d'être l'objet du regard avec la passivité, selon l'observation : l'homme regarde; la femme est regardée (Dyer 1992 : 198). Leurs yeux insolents, sévères, se posent sur le lecteur, ou, pour encore mieux communiquer le sentiment de l'impénétrabilité masculine, les yeux sont même invisibles derrière des lunettes de soleil ou sous la visière d'une casquette en cuir, mais on sait qu'ils se posent sur vous. Le visage n'est pas individualisé. La critique que Tom a entendue tout le long de sa carrière était que ses hommes se ressemblaient tous (Hooven 1993 : 88). Même quand Tom exécute des commandes individuelles, ce qui arrive de plus en plus pendant les années 1980, malgré une certaine spécificité pour plaire au client, le visage garde toujours un aspect prototypique. Cette qualité universelle contribuait en grande partie au succès international de l'artiste, n'importe quel lecteur pouvant s'imaginer dans le fantasme. Ramakers constate: «Ce corps uniforme, combiné avec le visage uniforme, contribuait beaucoup à la qualité iconique de l'œuvre : l'individualité fut neutralisée en faveur d'une seule image idéale ${ }^{23}$.» (Ramakers 2000: 69) Pour Nayland Blake, «la similitude [des hommes] nous fait nous sentir chez nous. C'est un monde que nous reconnaissons, mais sans les limites sur nos désirs ${ }^{24}{ }$ (Blake 1995 : 348).

11 La masculinité étant associée à l'activité, les hommes de Tom sont souvent en train de faire quelque chose. Leurs occupations étant "masculines ", le bûcheron tient une hache, le policier arrête le motard, le marin manœuvre un bateau, le docker prépare 
des balles de marchandises, le jeune motard met ses forces à l'épreuve dans une fête foraine. Même si deux hommes s'intéressent beaucoup plus l'un à l'autre qu'à une quelconque activité, une pelle, un balai, un fouet, une barre d'haltères, une casquette de motard par terre vont tous suggérer qu'ils étaient en train de faire quelque chose de masculin. Dans ses dessins, Tom s'intéresse aussi au réseau de gestes et de postures qu'il peut donner au corps, aux façons de se tenir et de bouger qui seront séduisantes. Jambes écartées, bras croisés ou poings sur les hanches, la pose est toujours ouverte, la démarche orgueilleuse ; adossé contre un mur, on fait le pied de grue, le pouce dans la ceinture ; se caressant pour diriger le regard vers le centre d'intérêt qu'est l'entrejambe bombé, on étale ce qu'on a à offrir. En fait, dans ses dessins en silhouette, ce n'est que la silhouette noire de l'homme qu'on voit se détacher sur le blanc du papier: tout est pose. Assis, les bras étirés le long d'un mur ou contre le dos d'une chaise, les jambes sont écartées d'une façon indolente pour mettre en relief la bosse. Tel un fauve au repos, le corps respire toujours la force et la puissance : il se dégage une impression d'énergie en réserve, l'impression que l'homme est prêt à se lever d'un bond pour passer à l'action sexuelle. Ce qui coïncide tout à fait avec la tradition de photos d'hommes destinées aux femmes où Dyer constate que le corps masculin, rarement allongé mais aux muscles tendus, est toujours posé pour suggérer le potentiel de l'action (Dyer 1992 : 202). Blake a noté aussi que les corps dessinés par Tom boudent. Il remarque que la moue est un signifiant sexuel puissant dans l'art occidental, et que chez Tom ce ne sont pas seulement les lèvres qui y contribuent, mais les yeux, le torse, même les pénis, qui sont tous pleins d'une énergie sexuelle voluptueuse : "C'est cette chair qui fait la moue, à travers les vêtements et de l'autre côté de la rue, qui produit l'air lourd de sexualité dans le monde de Tom ${ }^{25}$. » (Blake 1995 : 348)

\section{L'influence du corps}

12 Ramakers commente ainsi la grammaire corpographique de Tom: «Pénis en érection et indices tels que le mot Tom acquirent le statut de symboles de la masculinité gay à cause de leur emploi répété pendant de nombreuses années. Tom créa son propre langage de signes qui se détachait de plus en plus des codes existants pour former une grammaire autonome ${ }^{26}$.» (Ramakers $\left.2000: 177\right)$ C'est en apprenant cette grammaire par cœur et à force de la répéter que les admirateurs de Tom ont construit leur identité. En modifiant leur corps, en s'habillant, en posant et en bougeant selon les codes qu'ils voyaient dans ses dessins, ils ont joué un rôle qui, par la réitération d'actions et de gestes, les transformaient en «Tom's Men». L'artiste érotique contemporain de Tom, Étienne, note que très tôt les homosexuels ont commencé «à s'habiller et à se coiffer et à prendre les positions et les attitudes qui sont dans ses dessins ${ }^{27}$ ", toutes choses qui rappellent l'importance du caractère performatif dans la formation de l'identité selon Judith Butler. Elle écrit : «Le genre, c'est la stylisation répétée des corps, une série d'actes répétés à l'intérieur d'un cadre régulateur des plus rigide (sic), des actes qui se figent avec le temps de telle sorte qu'ils finissent par produire l'apparence de la substance, un genre naturel de l'être ${ }^{28}$.» (Butler 2005 : 109-110) «De tels actes, gestes, et accomplissements, au sens le plus général», continue-t-elle, "sont performatifs, par quoi il faut comprendre que l'essence ou l'identité qu'ils sont censés refléter sont des fabrications, élaborées et soutenues par des signes corporels et d'autres moyens discursifs ${ }^{29}$.» (Butler $\left.2005: 259\right)$ Si le performatif réussit, et Butler suggère que ce succès est toujours seulement provisionnel, c'est parce 
que « l'action fait écho à d'autres actions antérieures et accumule une force d'autorité à travers la répétition ou la citation d'une série de pratiques antérieures et autoritaires ${ }^{30}$ » (Butler 1993 : 227), qui, dans ce cas, seront les dessins originaux de Tom.

De nos jours, l'influence de Tom peut toujours se voir dans les profils sur des sites fétichistes où les hommes s'habillent selon les règles vestimentaires de ses figures et adoptent aussi des poses qui rappellent les postures de ses dessins. Dans n'importe quel bar « hard " aussi, les dessins de Tom seront collés au mur et les hommes prendront des poses qui rappellent ce qu'ils ont vu dans les dessins du Maître. Par l'image plutôt que par la parole, Tom a montré à une génération d'homosexuels euro-américains comment se comporter. Son œuvre constitue un manuel visuel d'étiquette. Au XVII ${ }^{e}$ siècle, à une époque où l'élite sociale se définissait de plus en plus par les manières et le goût, Faret offrait à son public L'Honnête Homme, ou l'Art de plaire à la court; pour son public spécialisé du $\mathrm{xx}^{\mathrm{e}}$ siècle, Tom a signé pour ainsi dire L'Homme selon Tom, ou l'Art de plaire dans le monde gay. Malgré lui, il proposait des modèles de comportement ${ }^{31}$. Ses images inspiraient ses lecteurs à imiter les corps et les poses qu'ils voyaient, lesquelles représentaient autant de signes pour d'autres hommes gay, des signes qu'on pouvait lire, interpréter, décoder et imiter. Avec le temps, les actions et les gestes répétés créaient l'illusion de la masculinité.

Tom fait tout ce qu'il peut avec sa matière première, le corps masculin, pour créer un objet à admirer, objet qui aura donc un effet sur un autre corps, celui du lecteur de ses images, et sur lui-même aussi (Tom savait qu'un dessin était réussi s'il bandait en le dessinant). Mais son influence va au-delà du frisson érotique. En virilisant le corps homosexuel, Tom a révolutionné la conception que quantité d'homosexuels avaient d'eux-mêmes, produit du fétiche de la catégorisation dont est férue la société hétéronormative. Grâce à lui, ils avaient une identité qui ne leur niait pas la masculinité. Et ils sont prêts à l'en remercier. Durk Dehner, qui était un ami de Tom aux États-Unis pendant les années 1980 et fondateur de la fondation Tom of Finland, dont le siège est à Los Angeles, affirme: "À cette époque, j'étais prêt à vivre ce qu'il représentait dans ses dessins. Très vite, il est devenu la personne qui a le plus influencé ma vie jusqu'à ce jour. Grâce à son œuvre, j'ai découvert que je n'étais pas moins homme que les hétérosexuels... » (Tom of Finland 1998 : 347). Un homme dans le film Daddy and the Muscle Academy se rappelle le moment où il a découvert les dessins de Tom. Américain, il travaillait à poser les voies pour le chemin de fer et dans les dessins de Tom il voyait la sorte d'homme qu'il trouvait attirant : « De voir deux ouvriers qui faisaient l'amour ensemble rendait cela plus acceptable pour moi. Étant d'un milieu ouvrier, jusqu'alors toutes les associations avaient été négatives. Cela me mettait plus à l'aise avec le fait que j'étais gay et que j'aime les hommes ${ }^{32}$. »

15 La découverte qu'on n'est pas seul marque une étape dans la vie de tout homosexuel. Pour Tom, les dessins de l'artiste érotique Quaintance furent une seconde découverte ${ }^{33}$. Ramakers décrit ce moment : "Ce ne fut rien moins qu'une révélation pour Tom. "Je n'en croyais pas mes yeux! s'exclama-t-il des années plus tard. Comment quelqu'un pouvait-il peindre de si beaux hommes avec un tel réalisme! Je voulais moi aussi parvenir à un résultat approchant !" » (Tom of Finland 1998 : 342) Les lecteurs de Tom se trouvent dans le même rapport avec ses images que Tom devant les dessins de Quaintance ou du héros d'Oscar Wilde, Dorian Gray, devant le « livre jaune ", c'est-àdire À Rebours de Huysmans : «C'était le livre le plus étrange qu'il eût jamais lu. [...] Des choses auxquelles il avait songé confusément lui étaient soudain présentes. D’autres, 
auxquelles il n'avait pas songé du tout, se révélaient à lui peu à peu ${ }^{34}$.» (Wilde 2001 : 173) En outre, l'image a un rôle central à jouer pour Dorian aussi. En regardant le portrait que Basil Hallward a fait de lui, Dorian affirme se reconnaître pour la première fois, et Ed Cohen remarque que son identification avec l'image réoriente Dorian vis-àvis de son identité et de son contexte social ${ }^{35}$. Edelman ajoute que Dorian maintient " une relation homographique avec son image peinte ${ }^{36}$ » (Edelman 2009: 150). Tout comme Dorian, les lecteurs de Tom se trouvent transformés par leur contact avec l'œuvre d'un artiste : tout à coup ils se découvrent, ils se comprennent. Dans les dessins de Tom, ses lecteurs participent à une nouvelle réalité où, tout en étant homosexuel, on n'a pas honte, on n'a pas peur, on vit sans doutes et sans confusion. Pour eux, les images individuelles de Tom, les petits livrets de bandes dessinées, les grands livres d'images sont autant de livres jaunes. Les lecteurs de Tom, séduits par son « monde gay de conte de fées" (Tom of Finland 1998: 346), veulent se transformer en homographes ${ }^{37}$ des hommes qu'ils voient dans ses dessins. C'est comme s'ils suivaient les conseils de Lord Henry qui parle à Dorian du besoin de se réaliser parfaitement. Et ils y ont réussi. Grâce au culturisme et à l'emploi des stéroïdes, un homme peut transformer son corps pour qu'il ressemble au corps d'un homme dessiné par Tom. L'artiste lui-même avait toujours dit : «Je ne cherche pas le réalisme, je veux exprimer nos fantasmes. ${ }^{38}$ » (dans Ramakers 2000 : 73) Mais à partir des années 1970, d'abord aux États-Unis et ensuite en Europe, les fantasmes corporels de Tom ont commencé à se transformer en réalité. Tom s'en inquiétait lui-même. En 1981, il témoigne de son inquiétude :

Le style macho est très à la mode. Aujourd'hui on voit beaucoup d'hommes qui sont le portrait tout craché du type que je dessine. Le culturisme peut vous faire parcourir une certaine distance dans cette direction. Je me demande de temps en temps s'il est bon que j'encourage cela. Il est difficile de dire ce qui est naturel et ce qui ne l'est pas. Mais de cette façon tout devient un peu exagérée ${ }^{39}$. (Ramakers 2000 : 12)

Tom apprend que tout créateur cède la propriété de ce qu'il a créé. Tom avait démontré que par le développement du corps, les homosexuels pouvaient récupérer la masculinité. Poussant cette masculinisation à l'extrême, ajoute Ramakers, « leur corps reconstruit devint hypermasculin, le phallus couvrant toute sa surface ${ }^{40} »(2000: 115)$.

\section{Le corps habillé}

17 En ce qui concerne l'œuvre de Tom of Finland, on ne saurait dissocier l'élément vestimentaire de son projet macho. Comme Tom le dit lui-même: "Pour moi, un homme habillé est plus érotique qu'un homme nu. Un homme nu est beau, bien sûr, mais habillez-le en cuir noir ou dans un uniforme - ah, alors il est plus que beau, il est sexy ${ }^{41}$.» (Hooven 1993 : 62) Shaun Cole renforce cette idée : «Comme tissu, le cuir noir est saturé de signification sexuelle. Il a des couches d'implications : le porter rehausse l'attrait sexuel par une indication de la force et du pouvoir ${ }^{42}$ " (Cole 2000: 111). "Atavique, pré-industriel et romantique ${ }^{43}$ " (Pat Califa, citée dans Cole $\left.2000: 115\right)$, le cuir est une étoffe masculine qui fait que celui qui le porte parait plus masculin. Dans l'après-guerre, cet intérêt a coïncidé avec la naissance de la figure du motard, symbolisé sur l'écran par un Marlon Brando ténébreux et dangereux dans L'Équipée sauvage (1953). Botté, chapeauté et ganté de cuir, en jeans, tee-shirt blanc et blouson de cuir noir, Brando incarnait, comme James Dean d'ailleurs, l'esprit de révolte qui 
caractérisait toute une génération de jeunes gens au début des années 1950. L'animalité du cuir, la virilité de Brando, la qualité phallique de la motocyclette constituaient un objet d'attirance fortement érotique non seulement pour Tom mais pour toute une génération d'homosexuels euro-américains. Pour Tom, l'essor du motard constituait un heureux hasard pour son projet de déstigmatiser l'homosexualité, de réinsuffler la virilité à des hommes comme lui qui se trouvaient condamnés par la société hétéronormative à une vie en dehors de la masculinité. Ramakers analyse ce phénomène: "En associant le fait d'être gay précisément avec ce symbole de la puissance et de la masculinité [qu'est le motard], Tom confirma ce que son public, à rebours de l'opinion courante, voulait croire de lui-même : vous êtes de vrais hommes, l'homosexualité est le zénith de la masculinitét4 " (Ramakers 2000: 134-135). Et il ajoute: «Le personnage du motard ouvre la voie à la figure la plus emblématique de l'œuvre de Tom : l'homme en cuir.» (Tom of Finland $1998: 346$ )

Mais tout aussi emblématique de son œuvre est le look qui dominait le monde gay des années 1970 et 1980 et qui n'a pas tout à fait disparu de nos jours : le clone. Dès sa première exposition à New York en 1980, Tom s'est vu attribuer la création du look clone. Dans sa critique de l'exposition, Robert Pierce écrivit :

Je vois le look très décrié du clone de Christopher Street - bottes de chantier, jeans Levis, chemise de bûcheron à carreaux, blouson de cuir-comme une sorte de réalisation du monde fantastique de Tom. Les hommes gays, par le pouvoir symbolique des vêtements, affirment l'image qu'ils ont d'eux-mêmes en tant qu'hommes. La fantaisie de Tom se réalise sur Christopher Street ${ }^{45}$... (Pierce 1980 : 13)

Encore une fois il y a eu appropriation des signes de la masculinité hégémonique, mais, comme Butler le signale, « les réitérations ne sont jamais simplement des répliques de l'original ${ }^{46} »$ (Butler 1993 : 226). Le corps étant toujours d'importance primordiale, les jeans, la chemise, le tee-shirt sont portés de façon si serrée que, tout comme dans les dessins de Tom où les contours des muscles, des fesses, et des parties sont tellement mis en relief par les vêtements que portent les hommes qu'on pourrait dire qu'ils constituent une seconde peau, ceux du clone révèlent le corps plutôt qu'ils ne le cachent $^{47}$. Au début des années 1980, quand la mode clone s'était élargie pour inclure les codes vestimentaires de l'ouvrier de chantier, Tom a toujours insisté sur l'importance du vêtement pour le projet de masculinisation: "Dans le passé, les homosexuels ont tous été classés comme faibles et sans caractère. Cette image est en train de changer grâce à ces modes macho ${ }^{48}$. » (cité dans Ramakers $2000: 134$ )

C'est également des années 1940 que date l'admiration de Tom pour les uniformes. C'est pendant la Seconde Guerre mondiale, quand Touko servait dans l'armée finlandaise, qu'il fait pour la première fois la connaissance de "vrais hommes »: militaires soit finlandais soit allemands soit russes, mais toujours en uniforme. Ce sera un look qui le marquera pour le reste de sa vie et qui, grâce à ses dessins, sera déterminant dans le monde homosexuel de l'avenir. Après l'armistice avec l'Allemagne en 1941, la Finlande accueille des soldats allemands dans leur uniforme conçu par l'homosexuel Hugo Boss, uniforme qui a même de nos jours des connotations d'autorité, de puissance et de discipline. Les bottes et le cuir étant essentiels au look nazi, il n'était pas difficile pour le jeune Touko d'érotiser l'uniforme militaire, de le considérer comme la quintessence de la virilité, comme un moyen de communiquer de façon visuelle ce que cela veut dire d'être un homme. En tant que tels, les uniformes joueront un rôle principal dans le projet de virilisation de l'homosexualité que s'est donné Tom. Critiqué pour avoir 
incorporé la croix gammée dans ses premiers dessins, Tom dessinera pendant le reste de sa carrière des uniformes génériques, n'appartenant à aucune nationalité spécifique. Ses militaires de rêve s'habilleront d'uniformes neutres quant à leur pays d'origine, qui sera Tomland, un monde gay utopique où l'uniforme de fantaisie se porte hypermoulant et emprunte des éléments à n'importe quel uniforme - à moins que ses éléments ne fassent tout simplement partie des codes de la masculinité, se prêtant ainsi à une charge érotique.

21 Pour Williams, les vêtements que portent les hommes de Tom sont «les véritables icônes de la masculinitét ${ }^{49}$ " (Williams 1989 : 35). Mais de plus en plus, ses motards et ses policiers sont plutôt des motards et des policiers de fantaisie, couverts de signes de la masculinité mais sans avoir un look cohérent. Toute cohérence disparait en effet sous des emprunts à n'importe quel look qui crie la masculinité. S'il y a un placard homosexuel, chez Tom, c'est le magasin d'accessoires virils d'où il tire complaisamment jodhpurs ou chaps, bottes de cavalier et éperons, baudriers et harnais, ceintures, cordons et lacets, bracelets et brassards cloutés, gants et gantelets, clés ou toute autre chose qui peut pendre de la taille. Encore une fois, par ses dessins, Tom donne l'exemple à ses lecteurs, qui se montraient prêts à le suivre en s'appropriant tout élément vestimentaire tiré du répertoire masculin à leur disposition et à faire preuve de créativité en les assemblant selon une sensibilité gay. Un homme dans le film Daddy admet : «Plus ces mecs portent de ces choses-là, plus je les aime ${ }^{50}$. " Qu'importe que le look soit créé à partir d'éléments composites tirés d'une variété de sources, que ce soit un look hybride donc, ce qui est important c'est que c'est un look masculin, dont tout homosexuel pourrait reconnaître la signification. En outre, puisque les éléments divers dont il est créé font tous partie de codes vestimentaires ultramasculins, c'est aussi un look qui passera parmi les hétérosexuels.

Un dessin surtout semble résumer l'idée d'hybridation : «L'Auto-stoppeur ». Dans Tom of Finland: The Art of Pleasure, ce dessin est choisi comme symbolique de l'œuvre de Tom pendant les années 1970, « les années des clones ». Torse nu, l'oreille percée, il porte un short en cuir, une ceinture avec une boucle "Tom ", des bottes de motard, un bracelet clouté. Il a un fouet dans une botte, un tatouage "Tom's Saloon" au bras droit, un flacon de poppers ${ }^{51}$ autour du cou, un bandana dans sa poche arrière droite et des menottes se balançant à la ceinture. Selon Bordo, dans ce dessin, « les symboles de la masculinité agressive [...] sont tellement surabondants qu'on ne peut pas les prendre au

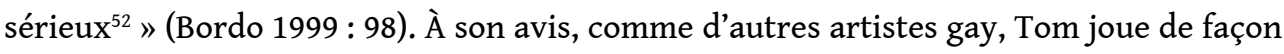
ironique avec «les icônes de la masculinité phallique ${ }^{53}$ » (100). Encombrer un homme de tant de symboles masculins sans fonction tourne à la parodie. D'où l'ironie de l'imitation par les hétérosexuels de telles modes vestimentaires et même du corps musclé. 


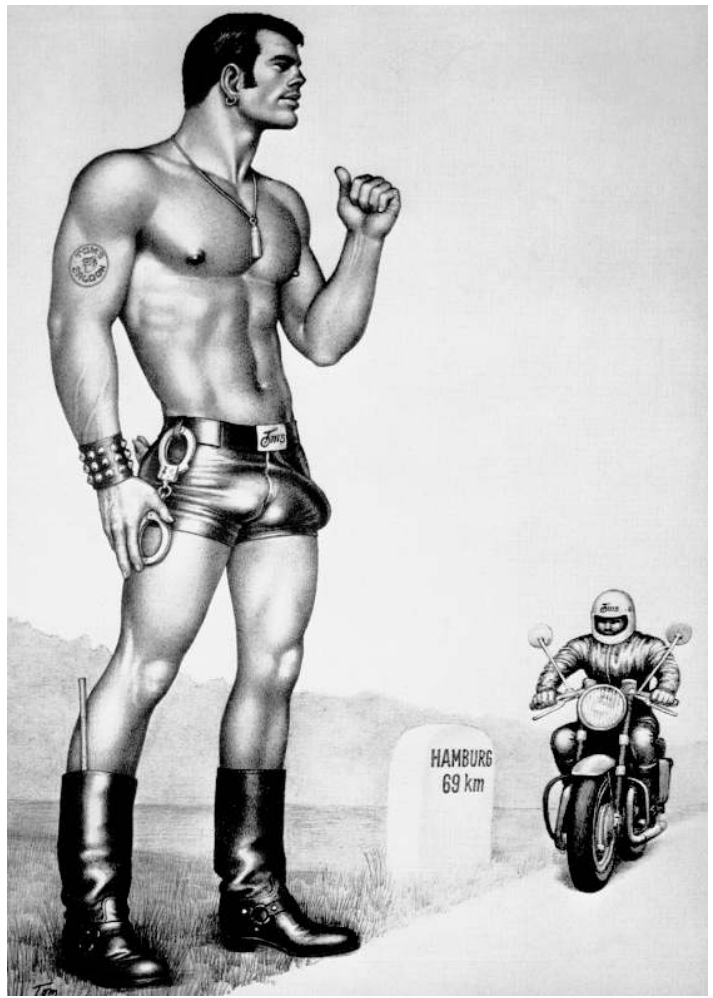

(c) Tom of Finland foundation, 2003

\section{Le corps subversif}

L'imitation de l'hypermasculinité par les homosexuels peut être perçue comme le travestissement macho. Butler affirme fortement qu'on juge la qualité d'un travestissement par sa vraisemblance. Le fait que le travestissement peut être lu par d'autres homosexuels indique qu'il n'est pas tout à fait parfait : on note les divergences entre la véritable masculinité et l'imitation, ce qui ne pose pas de problème pour les gays, le public original. Mais, grâce à une plus grande visibilité homosexuelle, se développa bientôt un second public, cette fois hétérosexuel et inattendu, mais attiré par la masculinité du look. Selon Butler, pour qu'un travestissement réussisse, il faut que la lecture soit « une sorte de vision transparente, où le paraitre et ce qu'il signifie coïncident ${ }^{54}$ " (Butler 1993 : 129). Pour les deux publics, le look clone réussissait : les homosexuels sachant qu'il s'agissait d'un travestissement, mais tout à fait contents du fait ; les hétérosexuels ne voyant que la masculinité apparente. Butler conclut - et, dans notre contexte, la première phrase pourrait s'appliquer aux homosexuels et la deuxième aux hétérosexuels :

Quand ce qui paraît et la façon dont on le «lit» s'écartent, l'artifice de la performance peut être lu comme artifice; l'idéal se sépare de son appropriation. Mais l'impossibilité de lire signifie que l'artifice a marché, l'approximation de la vraisemblance paraît avoir été atteinte, le corps performant et l'idéal performé ont l'air indistinguables ${ }^{55}$. (Butler $1993: 129$ )

Certes, on peut voir l'attrait pour les hétérosexuels du look clone, un look qui célèbre une masculinité prolétarienne et ses associations avec le travail physique et la grande 
nature ou la rébellion romantique du motard. On se trouve devant une sorte de mise en abyme: Tom s'inspire de la masculinité hétérosexuelle et l'exagère. Il se trouve imité par les homosexuels qui sont déjà bien loin maintenant de l'original. Ensuite les hétérosexuels, ne voyant que la masculinité du look, lisent cette mode macho portée par les homosexuels (qu'ils ne reconnaissent pas à cause de leur air macho récemment inventé) comme idéalisation de la masculinité hégémonique - ce qui est d'ailleurs vrai, mais sans se douter qu'elle trouve son origine chez un dessinateur érotique gay. Michael Bronski commente ainsi le phénomène : «Il devenait difficile, dans certains quartiers de certaines villes, de distinguer les hommes gay des véritables ouvriers de chantier, ou de distinguer les hommes hétérosexuels qui s'habillaient comme les homosexuels des homosexuels habillés comme les ouvriers de chantier ${ }^{56}$." (Bronski 1984 : 71)

Les images de Tom et l'homme gay à qui il a donné naissance brouillaient les pistes. En insufflant la masculinité à l'homme gay, il rendait le fait même que celui-ci était gay imperceptible à l'œil hétérosexuel qui n'arrivait ni à voir ni à lire les signes d'une sensibilité queer sous l'hyper-masculinité qui est le legs de Tom à l'homme gay. Avant Tom, l'homosexuel n'était pas menaçant parce qu'il était facilement écarté d'emblée de par sa visibilité. On le reconnaissait au caractère efféminé inscrit sur son corps, et, le reconnaissant, on pouvait l'exclure, le chasser, rire de lui. Dépourvu d'une telle visibilité, dans un corps masculin qu'on n'arrive pas à décoder, l'homosexuel pose une menace à la logique de l'hétérosexualité (Edelman 2009 : 145-146). Les hommes de Tom confondent les attentes. En faisant étalage de leur masculinité, en refusant d'être exclus d'un monde masculin, en se proclamant hommes, donc, les hommes de Tom exposent le côté arbitraire de la logique des représentations du monde hétérosexuel et ce faisant ils le déstabilisent. En fait, Tom « queerisait » la masculinité hétérosexuelle.

La société hétérosexuelle exige un "registre de la visibilitéf7» (Edelman 2009: 143).

Pour Tom, il s'agit aussi de la visibilité. Pour lui, l'homosexualité est un acte public. C'est que, là où la société hétérosexuelle s'attend à l'efféminé et à la passivité pour pouvoir facilement étiqueter un homme comme homosexuel, Tom déconcerte par la sorte de visibilité qu'il encourage. En offrant à la société hétérosexuelle des valeurs d'autorité et de puissance, il menace «la stabilité des paradigmes ${ }^{58} »$ (145). En confondant les masculinités hétérosexuelles et homosexuelles, Tom compromet la logique binaire que prise la société hétéronormative. Il bouleverse la perception de la similitude et de la différence, ébranlant la fiabilité du corps comme "garant d'identité sexuelle ${ }^{59}$ " (ibid), ce qui peut mettre en doute l'identité même des hétérosexuels. Certes les hétérosexuels peuvent condamner la masculinité prônée par Tom comme artificielle, mais ils ne sauraient nier que le corps masculin n'est plus leur domaine privilégié et qu'un corps musclé n'est plus une garantie d'hétérosexualité. Leur masculinité à eux n'est plus incontestée; pour différencier deux corps, il faut que le corps hétérosexuel aussi se soumette maintenant à la lecture. Autrefois c'était seulement le corps homosexuel qui devait souffrir le stigmate de la textualité, mais maintenant que le corps hétérosexuel et le corps homosexuel sont homographes l'un de l'autre, hétérosexuels et homosexuels se trouvent donc tous dans la même galère. Une telle ambiguïté est troublante pour les hétérosexuels qui se tiennent toujours à l'affût de différences identitaires. Si l'homographèse représente " une discipline de contrôle social ${ }^{60} »(147)$, l'élan que Tom a donné à la masculinisation homosexuelle peut être 
considéré en quelque sorte comme un acte de résistance foucaldienne contre la société hétéronormative, qui cherche toujours à classer les homosexuels par leur corps.

Néanmoins, un des buts des marqueurs de la virilité dite hétérosexuelle sur un homme gay est de plaire aux autres hommes gay. Voilà le paradoxe de la masculinité gay, selon Pronger, qui l'appelle un « ersatz de masculinité ${ }^{61}$ » (Pronger 1990 : 232). Grands, beaux, forts, de tels hommes ont l'air de participer au monde patriarcal, quand, au contraire, ce n'est qu'un style. Avoir l'air masculin dans le monde gay est un atout, mais derrière les symboles érotisés de la masculinité, ce n'est qu'ambiguïté et paradoxe. Pronger signale que «l'homoérotisme reflète un sens paradoxal de ce que cela veut dire d'être un homme ", le désir homoérotique représentant "une faim de la violation de la masculinité62 ${ }^{2}$ (Pronger 1990 : 71, 76). Les corps musclés des hommes de Tom sont des véhicules de subversion. L'apparence ne correspond pas à la réalité, l'illusion ne reflète pas la vérité, tout n'est que fumée et jeu de miroirs. Aussi de tels hommes exposent-ils comme arbitraire tout ce qui parait logique et immuable dans la présentation de soi. En ébranlant «la logique de l'identité comme régulation ${ }^{63}$ " (Edelman 2009: 147), ils sèment parmi leurs homologues hétérosexuels un certain doute sur la masculinité et un sentiment de sa fragilité. En révélant le gouffre qui peut exister entre l'hypermasculinité et l'hétérosexualité, ils dénaturalisent des associations qui paraissaient permanentes, universelles et naturelles. C'est pour des raisons de ce type que Pronger affirme : "La masculinité paradoxale a l'air masculin quand, en fait, si on la lit correctement à travers une lentille ironique, c'est la désintégration de la masculinitét $^{64}$ » (Pronger 1990 : 275). Même s'ils sont habillés en ouvriers de chantier, les hommes de Tom déconstruisent l'édifice hétéronormatif plutôt qu'ils ne contribuent à sa construction. Ils font réfléchir. Une interprétation butlerienne de l'élément parodique dans l'œuvre de Tom serait que son œuvre prouve que le « genre » n'est que parodie. Selon Butler, le travestissement n'est pas seulement une parodie de l'original, mais plutôt une parodie de la notion même de l'original, révélant que l'original qui est imité est «une imitation sans origine ${ }^{65}$ " (cité dans Sullivan 2003: 86). Ainsi "le travestissement "queerise" les notions essentialisées ou naturalisées de genre et de sexualité et du sujet qui sont parties intégrantes des discours et des institutions hégémoniques ${ }^{66}$ » (Ibid.). Ramakers le remarque également: "Le sexe gay est déjà transgressif à l'égard des normes sociales ${ }^{67}$ " (Ramakers 2000 : 222) Dans le film Daddy and the Muscle Academy, Tom fait un commentaire sur cette question de la norme et de la classification: "Je n'ai jamais voulu classifier les gens. D'après ma propre expérience, nous avons tous un peu de tout en nous, mais dans des proportions différentes. Rien n'est totalement étranger à personne. » Ce qui semble coïncider avec les idées de Foucault sur «l'art de la vie »: "Nouvelles formes de relations, nouvelles formes d'amour, nouvelles formes de création. Le sexe n'est pas une fatalité : c'est une possibilité pour la vie créatrice. ${ }^{68} »$ (Foucault 1996 : 382)

Par ses milliers de dessins, Tom a créé un monde où les homosexuels n'avaient pas peur, ne se cachaient pas, s'appropriaient pour eux-mêmes tous les symboles de la masculinité qui leur avaient été déniés et se montraient plus créateurs avec le matériel de base que leurs homologues hétérosexuels ne l'avaient jamais été, à tel point que vers la fin de sa carrière ce n'était pas seulement "Tom's Men » qui s'intéressaient à leur corps et s'habillaient de vêtements qui rappelaient les hommes de ses dessins. En encourageant la masculinisation du corps homosexuel, Tom a réussi dans son projet. Les hommes gay ne se considèrent plus comme exclus de la masculinité. Quantité d'homosexuels vont au gymnase, se maintiennent en forme et arborent un corps 
sculpté par l'exercice, mais les hétérosexuels cherchent toujours à lire le corps gay. L'efféminement n'a pas disparu, mais la réussite de Tom a été telle qu'un corps entretenu et musclé est aussi indicateur d'homosexualité. En outre, les muscles étant symboliques de la masculinité en général, les hétérosexuels se précipitent maintenant eux aussi vers le gymnase.

\section{BIBLIOGRAPHIE}

Arell, B. et Mustola, K., 2006, Tom of Finland. Ennennäkemätöntä - Unforeseen, Helsinki, Like.

Blake, N., 1995, « Tom of Finland : An Appreciation », dans C.K. Creekmur et A. Doty (dir.), Out in Culture: Gay, Lesbian, and Queer Essays on Popular Culture, Durham et Londres, Duke University Press.

Bordo, S., 1999, The Male Body: A New Look at Men in Public and Private, New York, Farrar, Straus and Giroux.

Bronski, M., 1984, Culture Clash: The Making of Gay Sensibility, Boston, South End Press.

Butler, J., 1990, Gender Trouble: Feminism and the Subversion of Identity, New York, Routledge. [2005, Trouble dans le genre, Cynthia Kraus (trad.), Paris, La Découverte.]

-, 1993, Bodies That Matter: On the Discursive Limits of Sex, New York, Routledge.

Cole, S., 2000, Don We Now Our Gay Apparel: Gay Mens Dress in the Twentieth Century, Oxford, Berg. Dutton, K. R., 1995, The Perfectible Body: The Western Ideal of Physical Development, Londres, Cassell. Dyer, R., 1992, « Don't Look Now: The Male Pin-Up », dans The Sexual Subject: A Screen Reader in Sexuality, London, Routledge, p. 265-276.

-, 1993, The Matter of Images: Essays on Representations, Londres et New York, Routledge.

Edelman, L., 1994, Homographesis: Essays in Gay Literary and Cultural Theory, New York, Routledge.

-, 2009, « Homographèse : identité corporelle et différence sexuelle », dans M.-A. Paveau et

P. Zoberman (dir.), Corpographèses : corps écrits, corps inscrits, p. 133-160.

Faret, N., 1631, L'Honnête Homme ou l'Art de plaire à la court, Paris, Du Bray.

Foucault, M., 1996, Foucault Live (Interviews, 1961-1984), New York, Semiotext(e).

Hooven, F. V., III, 1993, Tom of Finland: His Life and Times, New York, St Martin's Press.

Pierce, R. J., 1980, « The Case for Gay Art », Soho Weekly News, 6 février, p. 12-13.

Pronger, B., 1990, The Arena of Masculinity: Sports, Homosexuality and the Meaning of Sex, Toronto, University of Toronto Press.

Ramakers, M., 2000, Dirty Pictures: Tom of Finland, Masculinity, and Homosexuality, New York, St Martin's Press.

Reed, D., 1980, « Repression and Exaggeration: The Art of Tom of Finland », Christopher Street, avril 1980. 
Snaith, G., 2003, « Tom's Men. The Masculinization of Homosexuality and the Homosexualization of Masculinity at the End of the Twentieth Century », dans J. Still (dir.), Mens Bodies (Paragraph 26, 1-2), p. 77-88.

Sullivan, N., 2003, A Critical Introduction to Queer Theory, Édimbourg, Edinburgh University Press.

Tom of Finland [Touko Laaksonen], 1998, Tom of Finland. The Art of Pleasure [texte de Micha Ramakers], Burkhard Riemschneider (éd.), Cologne et New York, Taschen.

Wilde, O., 2001 [1890], The Picture of Dorian Gray, Donald L. Lawler (éd.), Norton, New York et Londres. [Le Portrait de Dorian Gray, V. Volkoff (trad.), Paris, Librairie Générale Française, coll. « Livre de Poche ».]

Williams, J., 1989, « Boots, Biceps, Bulges: The World of Tom of Finland », Drummer, 137, p. 32-37.

\section{Film}

Pohjola, L, 1991, Daddy and the Muscle Academy, Helsinki, Filmitakomo Oy.

\section{NOTES}

1. Touko Laaksonen (1920-1991) est le nom de l'artiste finlandais qui signait ses œuvres Tom of Finland. Voir la note 10.

2. C'est l'expression qu'il emploie pour désigner ses dessins. Sauf indication contraire, toutes les traductions sont de Guy Snaith.

3. "The necessity of "reading" the body as a signifier of sexual orientation » (Edelman $1994: 4$ ). La traduction française de ce chapitre figure dans le présent volume et les références des traductions des passages d'« Homographesis » y renvoient.

4. « A distinct, and therefore legible, anatomical code » (Edelman $1994: 5$ ).

5. «Its determining relationship to textuality and the legibility of signs» (Edelman $1994: 7$ ).

6. "The role of the stereotype is to make visible the invisible, so that there is no danger of it creeping up on us unawares. »

7. " we always have possibilities, there are always possibilities of changing the situation. »

8. Ses dessins des années 1940 montrent un monde de ce type, d'hommes en veston, aux petites écharpes, dansant ensemble.

9. «I wanted to make them as natural as possible. » Dans le film Daddy and the Muscle Academy [Papa et l'Académie du muscle] (Pohjola 1991).

10. C'est grâce à Bob Mizer qu'on connaît Touko Laaksonen sous le nom de Tom of Finland. Touko avait décidé que le prénom Tom était viril et sans prétention. Il signait donc déjà ses dessins « Tom », mais c'est Mizer qui ajouta of Finland, ce qui inquiétait un peu Tom parce qu'il craignait qu'on ne pensât qu'il représentait la Finlande en quelque position officielle. Ce qui n'était certainement pas le cas.

11. «Believing that a man could be strong, happy, manly AND homosexual, he began to formulate the prototype that has become the quintessence of gay masculinity the world over. » 12. "Soon I began to exaggerate their maleness on purpose to point out that ail gays don't necessarily need to be just "those damn queers," that they could be as handsome, strong, and masculine as any other men.»

13. " A photo-realistic approach »: Tom en parle dans le film Daddy and the Muscle Academy.

14. «A period of technical virtuosity »; « a precision which [...] is almost magical ».

15. Dans le film Daddy and the Muscle Academy. 
16. « Puissant symbols of masculinity » (Pronger $1990: 154)$.

17. "Muscularity is the sign of power-natural, achieved, phallic.»

18. "The phallus is the penis that takes one's breath away [...] because of its majesty. "

19. «A pictorial representation of the essence of manliness, a representation of the synthesis of every imaginable aspect of proper manhood.»

20. « The ultimate sign of masculinity. "

21. "This apotheosis of masculine symbols."

22. "The well-established symbolism that associates the hard, muscular body with the engorged, erect and potent phallus. »

23. "This uniform body, in combination with the uniform face, provided a great deal of the work's iconic qualifies: individuality was cancelled out in favor of one ideal image. »

24. "Their similarity makes us feel at home. This is a world we recognize, but without the boundaries on our desires. »

25. «It is this flesh that pouts, through clothes and across streets, that produces the heavy air of sex in Tom's world. »

26. "Erect penises and indices such as the word Tom acquired the status of symbols of gay masculinity because of their consistent use over many years. Tom created a language of his own that increasingly detached itself from existing codes to form an autonomous grammar. »

27. "They started to dress and comb their hair and take stances and attitudes that are in his drawings " [dans le film Daddy and the Muscle Academy]. Étienne était le nom d'artiste de l'Américain Dom Orejudos (1933-1991), artiste érotique gay et ami de Tom.

28. "Gender is the repeated stylization of the body, a set of repeated acts within a highly regulatory frame that congeal over time to produce the appearance of substance, of a naturel sort of being. " (Butler 1990 : 33)

29. "Such acts, gestures, enactments, generally construed, are performative in the sense that the essence or identity that they otherwise purport to express are fabrications manufactured and sustained through corporeal signs and other discursive means. » (Butler $1990: 136$ )

30. "That action echoes prior actions, and accumulates the force of authority through the repetition or citation of a prior, authoritative set of practices. »

31. En 1980, Tom insistait sur le fait que ses hommes n'étaient que des fantasmes, et tellement exagérés qu'ils ne pouvaient pas servir de modèles physiques ou de comportement (voir Reed 1980 : 20). Pour Tom, l'exagération ira de temps en temps jusqu'à la parodie (voir la discussion de "L'Auto-stoppeur", p. 121) mais ses admirateurs, bien que l'humour même ironique ne les dépasse pas, choisissent de voir et d'adopter tout ce qui est sexuel dans le look. Ceux qui n'ont pas le fétiche peuvent en rire.

32. «Seeing two blue collar men together enjoying each other made it more acceptable to me. From a blue collar background, up to this point all associations had been negative with it. It made me more comfortable with the fact that I am gay and that I like men. »

33. George Quaintance (1902-1957) fut un des pionniers de la peinture homoérotique aux ÉtatsUnis. Bob Mizer choisit une de ses œuvres pour la couverture de la première édition de Physique Pictorial.

34. «It was the strangest book that he had ever read. [...] Things that he had dimly dreamed of were suddenly made real to him. Things of which he had never dreamed were gradually revealed. » (Wilde 1988 : 97)

35. La discussion sur Dorian Gray se trouve dans Edelman $1994: 15-18$. Voir ici même, 149-153.

36. «A homographic relation to his painted image. » (Edelman $1994: 16$ )

37. Je reprends le terme d'Edelman, qui le définit comme « un mot qui présente la même forme écrite qu'un autre mais d'origine et de sens différents» (2009: 144). Il cite aussi une des définitions dans l'oxford English Dictionary: «identiques pour l'œil ». Le terme est courant en linguistique: un homographe est la forme graphique de l'homonyme c'est-à-dire une même 
graphie pour deux mots qui ont des sens différents, et éventuellement des prononciations différentes : vers/vers (nom et prépositions) ou couvent/couvent (verbe et nom).

38. "I'm not trying for realism. I want to express our fantasies. »

39. "The macho style is very fashionable, you see. These days you see many guys who are almost a spitting image of the type I draw. Bodybuilding can take you some way in that direction. I sometimes wonder whether it is good for me to encourage that. It's difficult to say what is natural and what isn't. But in this way it's all becoming a bit over the top. »

40. " Their reconstructed body became hypermasculine, the phallus covering its entire surface. " 41. "To me a fully dressed man is more erotic than a naked one. A naked man is, of course, beautiful, but dress him in black leather or a uniform-ah, then he is more than beautiful, then he is sexy! »

42. «As a fabric black leather is saturated with sexual meaning. It has layers of implication: the wearing of it enhances sexual desirability through an indication of strength and power.»

43. «Atavistic, pre-industrial and romantic. "

44. « By coupling gayness with precisely this symbol of potency and masculinity, Tom confirmed what his audience, against the grain of prevailing opinion, wanted to believe about itself: you are real men, homosexuality is the zenith of masculinity. "

45. «I see the much-maligned Christopher Street clone look-work boots, Levi's, plaid shirts, leather jackets as a kind of realization of Tom's fantasy world. Gay men, through the symbolic power of clothing, are asserting themselves as men. Tom's fantasy is being realized on Christopher Street. » À l'époque, Christopher Street était au centre du quartier gay de New York.

46. "Reiterations are never simply replicas of the same."

47. Sur l'exploitation de ce nouveau look gay par le monde commercial, voir Snaith 2003.

48. «In the past, homosexual men have always been typed as weak and characterless. That image is changing now thanks to those macho fashions. "

49. «Even the clothes these men wear are the very icons of maleness. »

50. "The more these guys have on of these things, the more I like them. »

51. Les «poppers" sont des ampoules de nitrite d'amyle, aux effets instantanés de stimulation, très répandus dans les années 1980 , en particulier dans les milieux gay.

52. "The symbols of aggressive masculinity [...] are so overabundant that they simply cannot be taken seriously. "

53. "The icons of phallic masculinity. »

54. " A kind of transparent seeing, where what appears and what it means coincide. »

55. "When what appears and how it is "read" diverge, the artifice of the performance can be read as artifice; the ideal splits off from its appropriation. But the impossibility of reading means that the artifice works, the approximation of realness appears to be achieved, the body performing and the ideal performed appear indistinguishable. »

56. "It became difficult, in certain parts of certain cities, to tell gay men from real construction workers, or straight men dressing like gay men from gay men dressing like straight construction workers. "

57. « Register of visibility. » (Edelman 1994 : 11)

58. «The stability of the paradigms. » (12)

59. «The guarantor of sexual identity. "

60. « A discipline of social control.» (13)

61. «Ersatz masculinity.»

62. "Homoeroticism reflects a paradoxical sense of what it means to be a man »; « a hunger for the violation of masculinity. »

63. "The logic of regulatory identity.»

64. «Paradoxical masculinity looks masculine when, in fact, if read correctly through an ironie lens, it is the disintegration of masculinity. » 
65. « An imitation without an origin » dans Gender Trouble, p. 138.

66. «Drag queers the essentialised or naturalised notions of gender, sexuality, and the subject that are integral to hegemonic discourses and institutions. »

67. "Gay sex is already transgressive of social norms. "

68. «New forms of relationships, new forms of love, new forms of creation. Sex is not a fatality : it's a possibility for creative life.»

\section{RÉSUMÉS}

L'artiste érotique finlandais Tom of Finland a contribué à la virilisation de l'homosexualité dans la seconde moitié $\mathrm{du} \mathrm{xx}^{\mathrm{e}}$ siècle. Prenant pour cible l'image de l'homosexuel efféminé, la stratégie centrale à la base du projet de virilisation de Tom était la représentation du corps masculin. En dessinant des images de gays avec des corps musclés, il a créé une image de la virilité qui a toujours été refusée aux homosexuels. Dès les années 1960, sa popularité lui a donné une énorme influence dans la création d'une identité gay masculine. L'accroissement de la visibilité des gays, bien bâtis et en tenue macho, signifiait que son influence s'étendait même de façon subversive au-delà du monde des gays. Le monde hétérosexuel n'est-il pas à présent déconcerté par le fait qu'avoir un beau corps n'est plus l'unique privilège des hommes hétérosexuels ?

The Finnish erotic artist Tom of Finland was instrumental in the masculinization of homosexuality in the latter half of the twentieth century. Targeting the image of the effeminate homosexual, the central strategy behind Tom's project of masculinization was the depiction of the male body. In drawing pictures of gay men with muscular bodies, he created an image of masculinity which had always been denied to homosexuals. From the 1960s onwards, his popularity gave him enormous influence in the creation of a masculine gay identity. The higher visibility of gay men, well built and in macho attire, meant that his influence even stretched subversively beyond the world of gay men. Is the straight world not now disconcerted that having a good body is no longer the unique privilege of heterosexual men?

\section{INDEX}

Keywords : homosexuality, masculinity, muscularity, strereotype, Tom of Finland

Mots-clés : homosexualité, masculinité, muscles, stéréotype, Tom of Finland

\section{AUTEUR}

\section{GUY SNAITH}

University of Liverpool 